\title{
Virulence and isozyme diversity of sexual versus asexual collections of Uromyces appendiculatus (bean rust fungus)
}

\author{
JAMES V. GROTH*, JOHN W. MCCAIN \& ALAN P. ROELFS $\dagger$ \\ Department of Plant Pathology, 495 Borlaug Hall, 1991 Upper Buford Circle, University of Minnesota, St. Paul, \\ Minnesota 55108 and †U.S.D.A.IA.R.S. Cereal Rust Laboratory, 1551 Lindig St., St. Paul, Minnesota 55108, U.S.A.
}

\begin{abstract}
Organisms that reproduce sexually have been assumed to be more genetically diverse (have more phenotypes with a more even frequency distribution) than those that are clonal. Rust fungi usually have regular sexual reproduction, but asexual species or populations are not uncommon. Two field collections of the bean rust fungus from sexually reproducing populations were compared with two collections from apparently nonsexual populations. Neither virulence on 10 differential lines of beans nor isozyme band patterns of five enzymes, as compared by the Gleason, Shannon, or Rogers indices of difference or with simple matching coefficients, supported the hypothesis of less population polymorphism in the asexual collections. The distribution of virulence differences among isolates in one of the asexual collections suggested that sexual reproduction was occurring at a low frequency which was, nevertheless, sufficient to maintain genetic variability.
\end{abstract}

Keywords: asexual/sexual diversity, bean rust, diversity indexes, genetic variability, Phaseolus vulgaris, Uromyces appendiculatus.

\section{Introduction}

Rust fungi are obligate parasites of higher plants. As such, they must rapidly produce or deploy new genotypes in response to changes in host resistance or movement to new host genotypes. Most organisms generate and maintain genetic diversity through sexual reproduction. Traditionally, asexual or obligately clonal organisms have been expected to be less genetically variable than sexual ones. Theoretically, clonal populations with fixed phenotypes that are subject to selection for extended periods should be less polymorphic than are sexual, outbreeding populations if no other forces maintain the polymorphism (Hartl \& Clark, 1989).

Sexual and asexual populations have been compared for relative levels of diversity within only a few species of plant pathogenic fungi. Several cereal rust fungi had sexual populations with more phenotypes based on virulence (called races) to a set of differentially resistant host lines than did asexual ones. Race frequencies in sexual populations were more evenly distributed as well. In asexual populations, one-few race(s) predominated, but abiotic and biotic environ-

*Correspondence. mental heterogeneity preserved a few rare races (Groth \& Roelfs, 1982). The population of the wheat stem rust fungus, Puccinia graminis forma specialis (f. sp.) tritici, from the north-western United States, where Berberis vulgaris - the host plant for the gametic stage of the rust - still grows, is more diverse for virulence (Roelfs \& Groth, 1980) and isozyme markers (Burdon \& Roelfs, 1985) than the asexual population of this rust in the Great Plains, where barberries have been eradicated. Similarly, Tooley et al. (1985) found greater isozyme variation in sexual populations of the potato late blight fungus, Phytophthora infestans. However, levels of diversity are higher in clonally maintained populations of higher plants, even where analogous combinations of sexual and clonal reproduction occur (Hamrick \& Godt, 1981; Ellstrand \& Roose, 1987).

Most of the comparisons cited above compare different, allopatric populations of different sizes, where the influence of environment on diversity of the population cannot be separated completely from the differences in frequency of sexual recombination. We used four small collections, all obtained from the northern Great Plains, of the bean rust fungus, Uromyces appendiculatus (Pers.:Pers.) Unger var. appendiculatus, to examine the hypothesis that sexual populations are more genetically polymorphic than asexual ones. Previous comparisons of isolates of the 
bean rust fungus have involved 1-2 isolates per collection site; differences in diversity among isolates within collections have not been examined.

Certain individuals of the bean rust fungus have lost their ability to produce teliospores, which function in this species not only to initiate sexual recombination but also as resistant structures to permit local overwintering or oversummering in a quiescent state. Asexual populations occur more often on snap bean; sexual populations occur mostly on dry bean types. These asexual populations of the fungus apparently survive in subtropical and tropical areas where green host tissue is available the year around, but they also regularly are transported to temperate areas. Gametic (pycnial) and zygotic (aecial) stages of this fungus have only been found in the northern United States, but this does not rule out local sexual populations having migrated from southern growing areas. It is also possible that asexual immigrants could grow near local sexual populations in a field and thus give a mixed collection (if truly asexual collections were distinguishable from truly sexual ones, e.g. by lower diversity for virulence, then mixed collections might be intermediate in distribution of such traits). We have found that selected asexual isolates differ in virulence and isozyme phenotypes (the sum of all virulences and isozymes determined) from sexual isolates of collections made in the north central states (Lu \& Groth, 1987; Linde et al., 1990b), but comparisons between collections have not been made.

Thus, the objective of this work was to compare two sexual with two asexual collections of the bean rust fungus for diversity among isolates within each collection. Representative collections of sexual (collections P14 and S9) and asexual (collections S3 and U2) life cycle types were maintained concurrently in the greenhouse and compared for diversity of isozymes and virulences to (mostly) single-gene differential host lines. Isozymes, the direct products of gene coding, can be easily obtained from germinated urediniospores of the bean rust fungus (McCain et al., 1992a) and interpreted readily because they are simply inherited as codominant alleles (Linde et al., 1989). Virulence markers are selectively and evolutionarily important because they can radically alter reproduction by the pathogen. A summary of this work was presented previously (McCain et al., 1992b).

\section{Materials and methods}

\section{Bean rust collections}

Four urediniospore collections of the bean rust fungus were made by aspirating all the spores from leaves gathered at single sites within single commercial bean fields or garden plots. Collection P14 was obtained in 1976 from Carrington, ND, on Phaseolus vulgaris L. cv. Pinto U.I. 111 ('P-111') beans, a dry bean cultivar that is highly susceptible to local bean rust populations (Groth \& Shrum, 1977); collection S9 in 1976 from Seafarer navy beans near Hector, MN; collection S3 in 1977 from Houston Co., MN, on a navy type bean; and collection U2 in 1977 from Elk River, MN, on a garden (snap) bean. All of the isolates from collections P14 and S9 have readily been induced to complete their sexual life cycles in the greenhouse at Saint Paul, MN Five isolates each from collections S3 and U2 had been tested - all failed to convert from uredinial production, i.e. they are apparently obligately asexual, at least under greenhouse growing conditions.

Urediniospores of the initial greenhouse generation had been collected in 1978-79 from 20 isolated uredinial pustules from each of the four collections, to provide sets of single-pustule (i.e. clonal, hence putatively genetically pure) isolates. The isolates were stored in a $-70^{\circ} \mathrm{C}$ ultralow freezer until the beginning of this study, when all 80 isolates were increased once on P-111 plants to produce a supply of fresh spores for virulence inoculations and for electrophoresis (some of the isozyme stains worked only with fresh, not previously frozen, spores - McCain et al., 1992a).

\section{Virulence inoculations}

Ten bean varieties were used as differentials to define rust virulence types. These 10 were selected because local collections of the bean rust fungus, when tested in previous studies, were polymorphic for virulence on these varieties (Linde et al., 1990b): Early Gallatin (snap bean), Golden Gate (wax snap), US \# 3 (green snap), \# 814 (small white), B-1349 (black), and five single-gene differential lines of small white beans: Ur1, Ur2, UrB, UrF and UrJ (Ballantyne, 1978). Seeds of the ten varieties of beans were germinated in steamed vermiculite in metal flats. After about 9 days, when the unifoliate leaves were half-expanded, the seedlings were transplanted to steamed soil in $15 \mathrm{~cm}$ clay pots.

Each set of inoculations consisted of three identical pots of transplanted bean seedlings, each pot containing one plant each of three of the differential varieties. Based on previous studies (e.g. Linde et al., 1990b), one of the differential varieties thought most likely to produce compatible reactions (B-1349, US \#3, or \# 814) was included in each set as an internal check. Six bean rust isolates were used per set, such that each set of inoculations replicated 18 host/isolate combinations. All 800 bean host/rust isolate combinations were repeated at least once. More than 100 sets of greenhouse inoculations were run, therefore, to test and verify all of the combinations. 
The plants were inoculated on the day of transplanting when the leaves were still half-expanded, the stage of maximum receptivity. About 1-2 $\mathrm{mg}$ of dry urediniospores were suspended in a few drops of Soltrol 170 light paraffin oil (Phillips Petroleum Corp., Bartlesville, OK) in a \# 00 gelatin capsule and lightly misted onto the upper side of the bean leaves. Stavely's 'confined-spray' method (1983) was adapted with a trigger-type spray head attached to an electric air pump at its lowest pressure setting, with the tubing attached to the outlet port. The spray head could then be held close to each primary leaf, wetting only a small portion of leaf surface at each instance. Three leaf positions were readily available on the cordate leaves: the tip and each of the two basal lobes. These three areas on each leaf were inoculated with three different isolates of the fungus. Because of the low spray pressure, no shielding of the leaf was necessary to prevent contamination between spots. Three other isolates were then inoculated on the opposite primary leaf. All six isolates were repeated on the other two plants in the same pot. Infection by other rust isolates on the same or opposite primary leaf had no effect on the reaction of any isolate tested.

After inoculation, the bean plants were incubated for $15-20 \mathrm{~h}$ at 100 per cent $\mathrm{RH}$ and then transferred to a $25^{\circ} \mathrm{C}$ greenhouse. Two weeks later, the infection type of the rust isolates on each host was scored by the 10point scale of Groth \& Shrum (1977).

\section{Electrophoresis}

Isozymes were resolved by polyacrylamide gel electrophoresis (PAGE), in vertical slab gels, from total protein extracts obtained by hand-grinding of germinated urediniospores in phosphate buffer, as described previously (McCain et al., 1992a). Distance of migration (relative mobility, 'Rf') of bands was standardized as the distance from the origin compared to the distance travelled by a tracking dye $(0.01$ per cent brom-
Fig. 1 Summary diagram of all 22 isozyme bands seen on polyacrylamide gels for five enzymes. $\mathrm{Rf}=$ relative mobility of bands on the gel, as per cent of the distance migrated by a bromophenol blue standard $(\mathrm{Rf}=100$ per cent). Roman numerals indicate putative loci (band clusters), separated by dashed lines; Arabic numerals indicate pattern number - number in parentheses is the number of isolates with this pattern. The pattern marked with an * was exhibited by the comparison isolate W73-2.

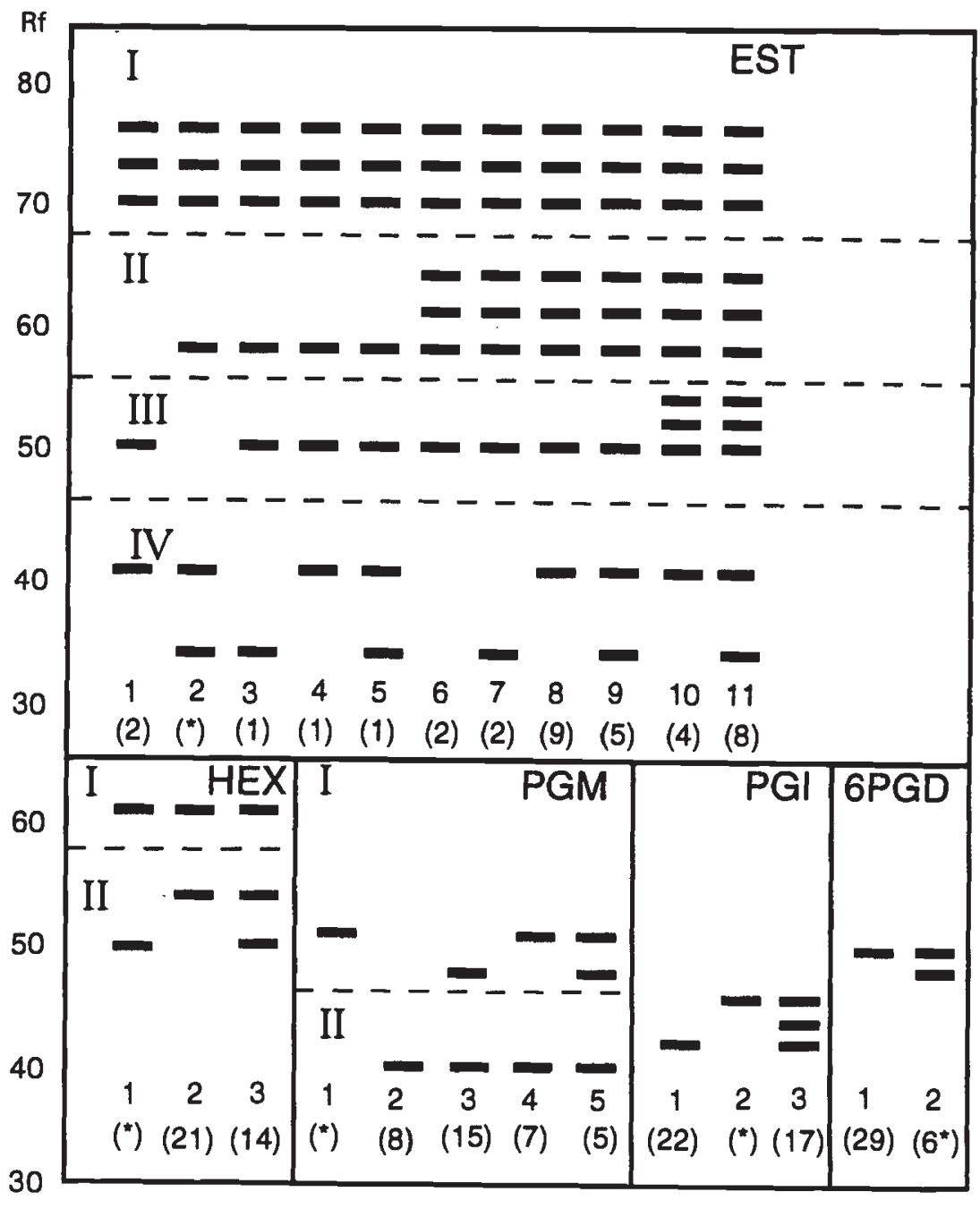

(c) The Genetical Society of Great Britain, Heredity, 75, 234-242. 
ophenol blue in 5 per cent Ficoll, $R f=100$ ). Five enzymes were chosen for which some rust collections were polymorphic in previous studies ( $\mathrm{Lu} \mathrm{\&} \mathrm{Groth,}$ 1987): arylesterase (EC 3.1.1.2., 'EST'), hexokinase (EC 2.7.1.1., 'HEX'), phosphoglucose isomerase (EC 5.3.1.9., 'PGI'), phosphoglucomutase (EC 2.7.5.1., 'PGM'), and 6-phosphogluconate dehydrogenase (EC 1.1.1.44., '6-PGD'). In PAGE gels, bean rust preparations produced bands grouped as four putative loci (markers) when stained for EST, two markers each for HEX and PGM, and one marker each for PGI and 6-PGD. A compilation of all the bands produced by one or more of the isolates is shown in Fig. 1. A control isolate, denoted W73-2, which is known to express the uncommon isozymes, was added to each gel as a comparison (McCain et al., 1992a). Sufficient spores were produced in the greenhouse increases to determine isozyme phonotypes for six isolates from collection P14, 11 from collection $\mathrm{S} 9$, seven from collection $\mathrm{S} 3$, and 11 from collection $U 2$.

\section{Diversity indices}

For calculating the Gleason, Shannon, and Rogers indices, the virulence scores for the rust isolates were simplified to A (avirulent; 0, 1, or 2 on the Groth/ Shrum scale), I (moderately virulent; 3-4 on the Groth/ Shrum scale), or V (virulent; 5-9 on the Groth/Shrum scale). Although the isozyme markers have been interpreted genetically (McCain et al., 1992a, supported in part by crosses made by Linde et al., 1989), in this study the 10 markers were scored simply as 1 (alike in number and gel location of bands produced) or 0 (different). Gleason, Rogers and Shannon indices of diversity were calculated as in Chong \& Kolmer (1993) and Groth \& Roelfs (1987).

The number of similarities of each isolate with every other isolate pairwise was summed to calculate simple matching coefficients (SMCs) (Linde et al., 1990b). Isolates were considered to have identical virulence scores if their scores on the Groth \& Shrum scale (1977) were (a) the same and four or less or (b) not more than two rating units different if the scores were five or higher (Linde et al., 1990b). Scores of 0 to 4 often indicate action of different resistance genes in the host in combination with corresponding avirulence genes in the pathogen. Therefore, isolates that induced different reaction types in the range of $0-4$ were not considered to express the same avirulence alleles, even though the reactions were all considered to be resistant type reactions. SMCs for isozyme markers were calculated as $\mathrm{SMC}=$ (number of markers in common) $/($ total number of markers).

\section{Results}

\section{Within-collection variation}

Virulence Collections P14, S9 (sexual), S3, and U2 (asexual) were polymorphic for virulence on eight, seven, six and nine of the 10 bean differential lines, respectively. The virulence phenotype (race identity) of an isolate is the 10 combined virulence scores for that isolate. One asexual collection (U2) was similar to the two sexual collections in the number and identity of their virulence combinations, but asexual collection S3
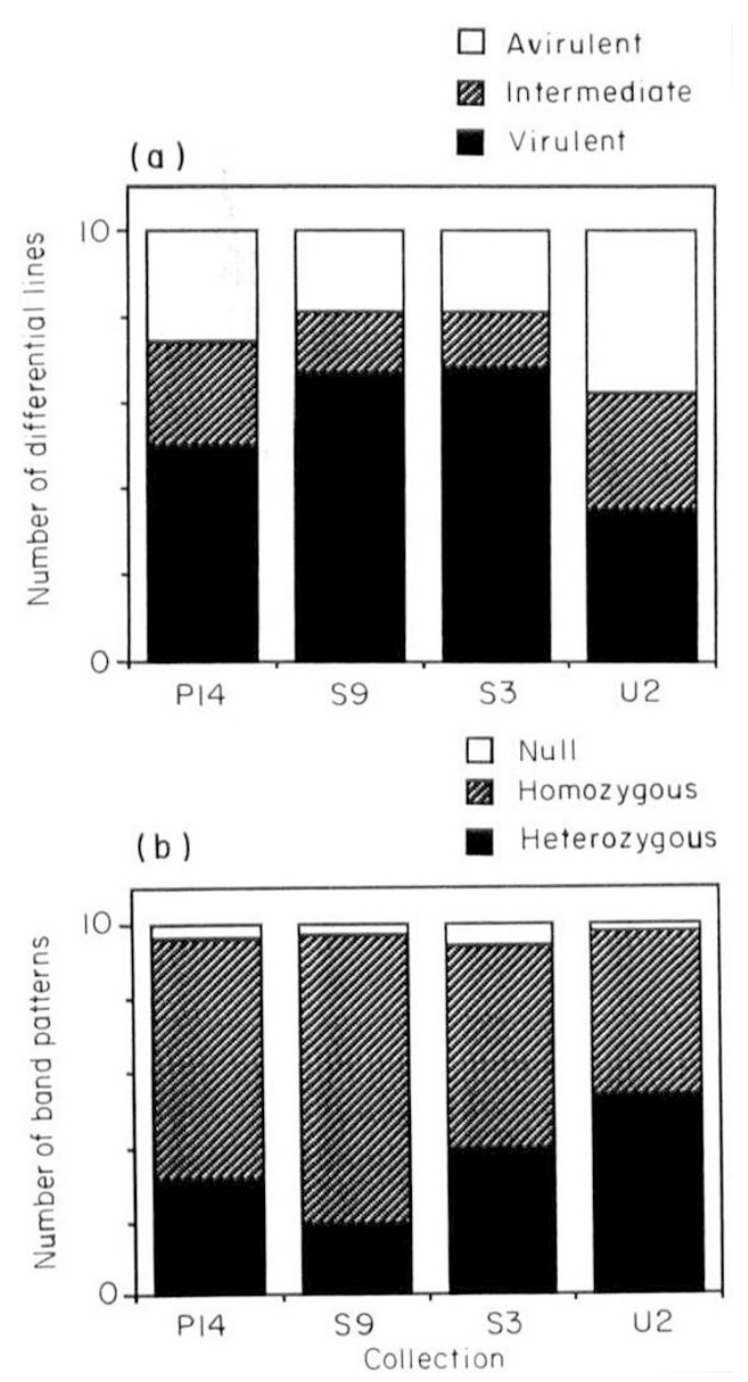

Fig. 2 Histograms of (a) mean proportion of isolates avirulent, intermediate (moderately virulent), and virulent on 10 bean differential lines and (b) mean proportion of null, homozygous, and heterozygous-type patterns for 10 isozyme loci expressed by isolates from two sexual (telia-forming) and two asexual (telia infrequent or absent) collections of Uromyces appendiculatus. Collections P14 and S9 are sexual; collections $\mathrm{S} 3$ and $\mathrm{U} 2$ are asexual. 
comprised half as many virulence phenotypes (races) as the other collections $(0.5$ phenotypes per isolate tested, versus $0.95,0.75$, and 1.0 for collections P14, S9, and $\mathrm{U} 2$, respectively). Virulence on most differentials was expressed by half as many isolates in collection $\mathrm{U} 2$ as in the other collections (Fig. 2a).

Isozymes The two sexual collections were polymorphic for alleles at five of the 10 putative isozyme loci. The two asexual collections were polymorphic for alleles at the same five loci as well as at two other isozyme loci. Of 21 total isozyme phenotypes (sum of bands produced in all markers for all enzymes), 19 occurred in only one or two isolates, one was exhibited by four isolates, and one by eight (five of these were in the $\mathrm{S} 9$ series).

The sexual isolates produced more isozyme band combinations for four of the enzyme markers than did the asexual ones. However, only the asexual collections exhibited both one- and two-band patterns for 6-PGD. Sexual collection S9 yielded the lowest ratio of electrophoretic phenotypes/isolate (P14-0.8, S9-0.5, S3-0.9, $\mathrm{U} 2-0.7)$.

Both asexual collections had more of the complex isozyme patterns that suggest heterozygosity (Linde et al., 1990a; McCain et al., 1992a) (Table 1, Fig. 2b). Frequencies of those bands that define heterozygous patterns were usually higher for the two asexual collections (Table 2); most other bands were fixed or nearly so in all four collections. The frequency of heterozygosity (sum of the heterozygous loci divided by the number of loci-Ayala, 1982) was $0.31,0.14,0.43$, and 0.60 for the isolates of $\mathrm{P} 14, \mathrm{~S} 9, \mathrm{~S} 3$, and $\mathrm{U} 2$, respectively. Locus heterozygosity for the seven variable isozyme loci (number of isolates with a given locus heterozygous divided by the number of isolates) ranged
Table 2 Frequencies of bands in complex isozyme markers (putatively heterozygous loci) from five enzymes in two sexual and two asexual collections of the bean rust fungus

\begin{tabular}{|c|c|c|c|c|}
\hline \multirow[b]{3}{*}{ Enzyme band } & \multicolumn{4}{|c|}{ Collection } \\
\hline & \multicolumn{2}{|c|}{ Sexual } & \multicolumn{2}{|c|}{ Asexual } \\
\hline & P14 & S9 & S3 & $\mathrm{U} 2$ \\
\hline EST-III $a \& b$ & 0.14 & 0.00 & 0.71 & 0.50 \\
\hline EST-IV $a$ & 0.86 & 0.47 & 1.00 & 1.00 \\
\hline$b$ & 0.57 & 0.13 & 0.29 & 0.80 \\
\hline HEX-II $b$ & 0.14 & 0.17 & 0.38 & 0.87 \\
\hline PGI-I $a \& b$ & 0.29 & 0.08 & 0.25 & 0.80 \\
\hline PGM-I $a$ & 0.14 & 0.07 & 0.38 & 0.77 \\
\hline$b$ & 0.43 & 0.93 & 0.38 & 0.46 \\
\hline
\end{tabular}

EST $=$ arylesterase HEX $=$ hexokinase;

PGI = phosphoglucose isomerase;

PGM = phosphoglucomutase. Roman numerals indicate marker (locus) number; lower case arabic letters denote putative alleles.

from 0 (no isolate apparently heterozygous) to 1 (all isolates heterozygous).

Indices of diversity Figure 3 presents three different indices of diversity within each of the four collections. These are expressed as the proportion of the mean of the four collections in each graph, to standardize the scale for the three indices.

Higher values for the Gleason index indicate more phenotypes, regardless of their relative frequency (Chong \& Kolmer, 1993). This index does not support the hypothesis, either for virulence or for isozymes, that more phenotypes would be found in the two sexual collections. Virulence (Fig. 3a) phenotypes were more

Table 1 Frequencies of complex (and putatively heterozygous) band patterns of five isozymes for two sexual and two asexual collections of the bean rust fungus

\begin{tabular}{|c|c|c|c|c|c|c|c|c|}
\hline & \multicolumn{5}{|c|}{ Enzyme } & \multicolumn{3}{|c|}{ Total } \\
\hline & EST & HEX & PGI & PGM & 6-PGD & Col & ection & Weighted \\
\hline Sexual collections & 0.77 & 0.18 & 0.18 & 0.06 & 0.00 & $\begin{array}{l}\mathrm{P} 14 \\
\text { S9 }\end{array}$ & $\begin{array}{l}0.32 \\
0.20\end{array}$ & 0.24 \\
\hline Asexual collections & 0.95 & 0.56 & 0.56 & 0.22 & 0.33 & $\begin{array}{l}\text { S3 } \\
\text { U2 }\end{array}$ & $\begin{array}{l}0.40 \\
0.54\end{array}$ & 0.48 \\
\hline
\end{tabular}

EST $=$ esterase; $\mathrm{HEX}=$ hexokinase $\mathrm{PGI}=$ = phosphoglucose isomerase;

$\mathrm{PGM}=$ phosphoglucomutase; 6 -PGD $=6$-phosphogluconate dehydrogenase.

Weighted score is the mean frequency of complex patterns adjusted for the different numbers of isolates tested per collection ( 6 from P14, 11 from S9, 7 from S3, 11 from U2). 


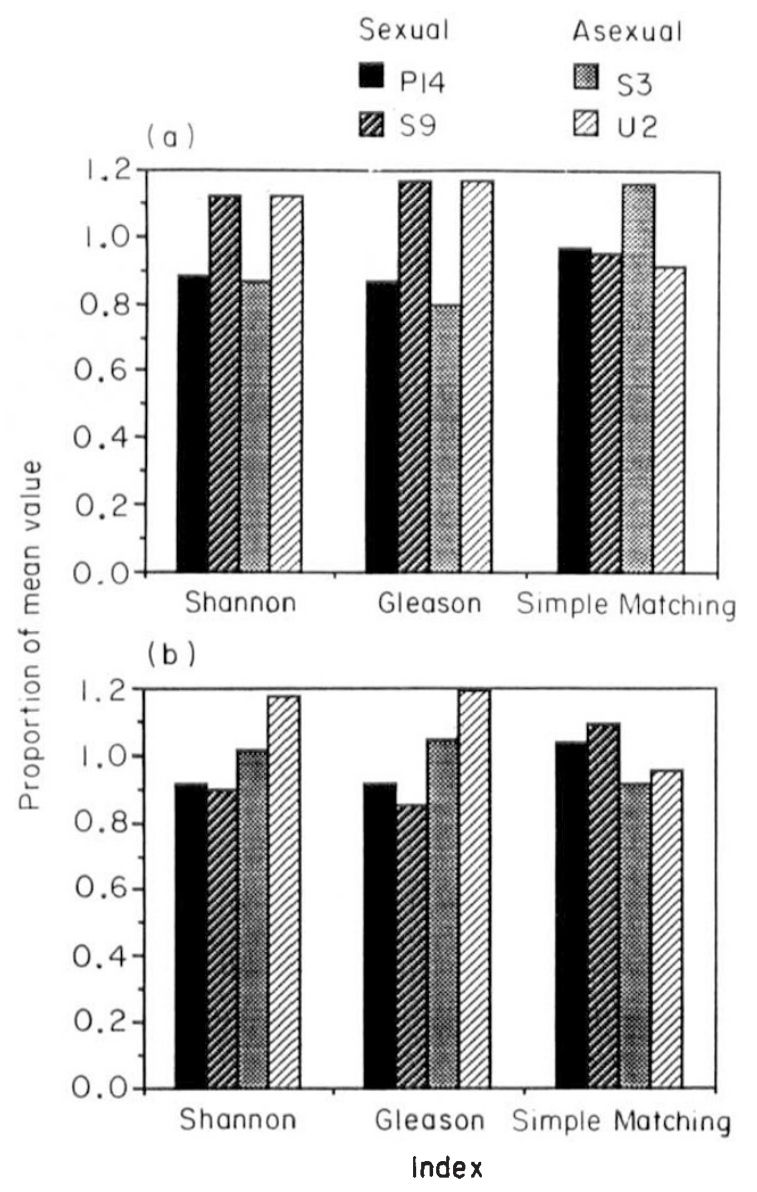

Fig. 3 Three indices of phenotypic diversity from two sexual (P14 and S9) and two asexual (S3 and U2) collections of Uromyces appendiculatus for (a) virulence on ten differential bean lines and (b) isozyme band patterns for five enzymes. Vertical scale shows proportion of mean of the four collections for each index.

numerous in collections S9 (sexual) and U2 (asexual). Isozyme (Fig. 3b) phenotypes were most numerous in collections S3 and U2 (both asexual), contrary to our expectations.

The Shannon index factors the frequency of each race with the total number of races: a high score indicates numerous and evenly distributed races. The pattern for this index was similar to that for the Gleason index but, for virulence, the Shannon index was significantly greater $(P=0.05)$ for collections $\mathrm{S} 9$ and U2 than for P14 and S3. For isozyme phenotypes, the Shannon index for collection U2 was significantly greater than for those of P14 and S9, but not so for S3 (other comparisons were nonsignificant).

The simple matching coefficients (SMCs) constitute a similarity index which estimates the mean phenotypic distance between all isolates (including those that are identical) within a collection. The higher the SMC, the

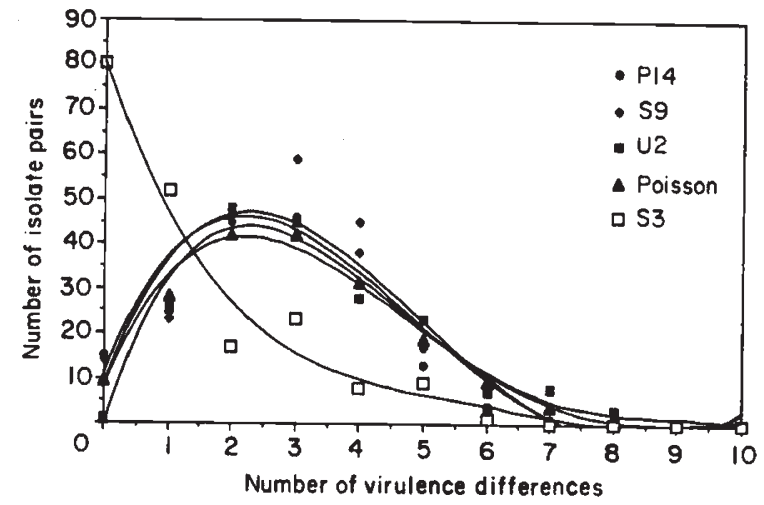

Fig. 4 Frequency distribution and fourth order fitted curves of number of virulence differences on 10 differential bean lines for all pairwise comparisons of 20 isolates from each of two sexual (P14 and S9) and two asexual (S3 and U2) collections of Uromyces appendiculatus. A Poisson distribution based on the common mean number of differences for collections P14, S9, and U2 is also plotted.

greater the intrapopulation uniformity. For virulence S3 had a higher SMC than the other three collections, but for isozymes the two sexual collections had higher SMC values than the two asexual collections.

When all isolate pairs were compared with all others, only one pair was found that differed in all 10 virulence scores and only 10 pairs of isolates differed in all seven of the variable isozymes. Ten of these 11 pairs included an isolate from the U2 series matched with one of the sexual isolates; the other matched S314 with a sexual isolate.

The distribution of the number of virulence differences (from none to being different on all 10 bean lines) between all pairs of isolates in each collection is shown in Fig. 4. The two sexual collections and U2 of the asexual collections all showed a continuous distribution of differences with a more-or-less central mode, suggesting that virulences were distributed randomly among isolates. Linde et al. (1990a) had previously found no linkage disequilibria in isozyme loci of the bean rust fungus. This randomness was confirmed by plotting an expected Poisson distribution from the mean number of differences in the three distributions, which gave a curve that was similar to those of three of the collections (Fig. 4). Asexual collection S3 had a distribution of virulences among isolates that had a mode of zero and, consequently, a lower mean number of differences (i.e. skewed towards fixation of one virulence phenotype) because more of the $S 3$ isolates were identical to one another than for the other three collections. Similar plots for isozyme differences were not useful because of the smaller sample sizes, especially for P14 and S3. 
Table 3 The Rogers index of difference among isolates in all possible comparisons of two sexual and two asexual populations of the bean rust fungus

\begin{tabular}{llllll}
\hline & \multicolumn{4}{c}{ Collection } \\
\cline { 2 - 3 } & \multicolumn{2}{c}{ Sexual } & & \multicolumn{2}{c}{ Asexual } \\
\cline { 2 - 3 } \cline { 5 - 6 } Collection & \multicolumn{1}{c}{ P14 } & S9 & & S3 & U2 \\
\hline U2 & 0.742 & 0.909 & & 0.857 & - \\
& 1.0 & 1.0 & & 1.0 & - \\
S3 & 0.857 & 1.0 & & - & - \\
& 1.0 & 0.95 & & - & - \\
S9 & 0.667 & - & & - & - \\
& 0.9 & - & & - & - \\
Mean & 0.755 & 0.858 & 0.905 & 0.836 \\
& 0.966 & 0.950 & 0.983 & 1.0 \\
\hline
\end{tabular}

Upper figure is for isozyme bands at 10 loci; lower for virulence on 10 bean differential lines.

\section{Between-collection variation}

Rogers index of difference The Rogers index of difference indicates the degree of distance between two samples based on differences in frequencies of all phenotypes found in either of them. Table 3 presents this index for the samples of the four collections, for virulence and isozymes. Most of the values for the Rogers index are very high (1.0 maximum possible, meaning no virulence phenotypes in common with any of the other collections), indicating little similarity among the collections. The lowest value of the Rogers index was obtained, for both kinds of markers, between the sexual collections P14 and S9. The two asexual populations were not similar for either kind of marker and, in fact, had no phenotypes in common for isozymes. Comparisons of sexual with asexual collections showed large phenotypic differences, except for P14 versus $\mathrm{U} 2$ for virulence.

Intercollection SMCs Likewise, SMCs can be compared, where each individual marker is summed over all comparisons of isolates between collections. As for the Rogers index, the two sexual collections, P14 and S9, were most similar, having the highest SMC for both kinds of markers. The two asexual collections, S3 and U2, were not alike, having the lowest SMC for virulence and the next-to-lowest for isozymes. In general, U2 was the most disparate collection, having the lowest average SMC value for both markers and the highest mean Rogers index of difference for virulence (1.0). Many of the differences presented here are small and cannot be verified statistically.

\section{Discussion}

Our hypothesis was that small, local collections of $U$. appendiculatus that are apparently asexual contain fewer phenotypes than do similar collections that reproduce sexually (Groth \& Alexander, 1989). This hypothesis was not supported by our data. The magnitudes of difference found in the comparisons made by Groth \& Roelfs (1987) of sexual vs. asexual collections of Puccinia graminis $\mathrm{f}$. sp. tritici were not found in the bean rust fungus. Sample sizes in the present study were small; nonetheless, they were large enough to reveal if the asexual collections were composed of a few clonally reproducing isolates. The variation among the isolates within each collection was exceptional, both for virulence and isozymes. Polymorphisms in sexual collections could result either from polymorphic or heterozygous local initial inoculum or from mixing of genotypes that were generated at different sites, where the initial inoculum at the single sites was not necessarily polymorphic.

One of the asexual collections (U2) differed from the other one and resembled the two sexual collections in the distribution of virulence differences among the isolates within each collection (Fig. 4). Previously, in studies of series of single isolates from several collections, Lu \& Groth (1987) found that one U2 isolate (U2-1) was more similar in isozymes to the isolates from their other asexual collections, but Linde et al. (1990b) found that another isolate (U2-12) was similar for both isozymes and virulence to their sexual isolates (by SMCs in the present study, U2-12 was more representative of the U2 series than was U2-1). Consequently, we re-examined collection $\mathrm{U} 2$ for ability to produce teliospores and found teliospores in very old pustules of a mass increase of $\mathrm{U} 2$ in the greenhouse but at lower levels than in average sexual isolates (J. V. Groth, unpublished). The distribution of virulence differences in the original collection suggests that these telia are functional in the field, and that the source of this collection was locally overwintering telia similar to the two sexual collections. Collection S3 contains only isolates that lack telia-producing ability. This population must have moved north from some unknown southern inoculum source. Its virulence and isozyme diversity indicate a large founder population either during the southern overwintering or in the reestablishment of the disease in the north.

That the bean rust fungus is able to maintain significant diversity in asexual populations may help explain the frequent occurrence of asexual forms in other rust fungi. Numerous rust species persist even in the absence of their gametic (pycnial) and zygotic (aecial) stages (e.g. Puccinia graminis where barberries have been removed) or apparently lack such stages (e.g. 
Hemileia vastatrix on coffee trees); yet these rusts may possess significant genetic diversity, e.g. the $>30$ known virulence races of coffee rust (Rodriguez et al., 1975).

Why are bean rust sexual populations not more diverse than the asexual populations? The asexual populations may have a high level of diversity compared to other fungi. Because of the potential for this rust fungus to overwinter as telia, farmers in Minnesota are advised to rotate their beans with other crops in order to break the sexual cycle. From our observations of the earliness and nonrandom patterns of initial infection in commercial dry bean fields and in our uninoculated plots (Groth et al., 1993) and on observations of aecia in other northern bean production areas (Schwartz et al., 1990), we think that the sexual populations are derived from basidiospore infection. But because our collections are made late in the season in order to obtain good samples, diversity that is present early may have eroded during the summer because less fit races were eliminated by selection, as has been shown with the crown rust pathogen of oats (Al-Kherb et al., 1987). In addition, bottlenecks may occur if only small numbers of inoculated basidiospores initiate the sampled population. The surprising finding is that there are several races in the asexual population, S3. If this population arose from urediniospores that were blown north, the numbers and diversity of these spores must have been large. Asexual isolates are not commonly encountered by us in Minnesota, and when they are blown into the state, weather conditions may be such that large numbers of urediniospores are so affected, as was postulated for the aphid vectors of Maize Dwarf Mosaic Virus (Berger et al., 1987). Two other contributors to the diversity of S3 that we do not think are as likely are (a) that the population is a composite of several migrations - such events are relatively rare - and (b) that sexual or parasexual recombination somehow occurred in the immediate past, neither of which events we can observe in the present population. We have manipulated several asexual isolates of this fungus for years, including one from S3. They appear to be stably asexual. Parasexual recombination is normally considered to occur at much lower levels than sexual recombination (Burnett, 1976).

\section{Acknowledgements}

We thank D. K. Adair, B. A. Haile, and L. Peterson for help in the greenhouse, and A. R. Pierce for both greenhouse and electrophoresis assistance. Rust collection P14 was obtained by J. Venette, and collection S9 from B. Mogen. Published as paper no. 20869 of the contribution series of the Minnesota Agricultural Experiment Station based on research conducted under Project 22-73, supported in part by USDA Competitive Grant no. USDA-58-5759-6-1 funds. Mention of a trademark name, proprietary product, or vendor does not constitute a guarantee or warranty of the product by the USDA and does not imply its approval to the exclusion of other products or vendors that also may be suitable.

\section{References}

AL-KHERB, S. M., ROELFS, A. P. AND GROTH, J. V. 1987. Diversity for virulence in a sexually reproducing population of Puccinia coronata. Can. J. Bot., 65, 994-998.

AYAlA, F. J. 1982. Population and Evolutionary Genetics: A Primer. Benjamin/Cummings, Menlo Park, CA.

Ballantyne, B. J. 1978. The Genetic Basis of Resistance to Rust, Caused by Uromyces appendiculatus in Bean (Phaseolus vulgaris). Ph.D. Thesis, University of Sydney.

BERGER, P. H., ZEYEN, R. J. AND GROTH, J. V. 1987. A phid retention of maize dwarf mosaic virus (potyvirus): epidemiological implications. Ann. appl. Biol., 111, 337-344.

BURDON, J. J. AND ROELFS, A. P. 1985. The effect of sexual and asexual reproduction on the isozyme structure of populations of Puccinia graminis. Phytopathology, 75, 1068-1073.

BURNETT, J. H. 1976. Mycogenetics. Wiley, London.

CHONG, J. AND KOLMER, J. A. 1993. Virulence dynamics and phenotypic diversity of Puccinia coronata f. sp. avenae in Canada from 1974 to 1990. Can. J. Bot., 71, 248-255.

Ellstrand, N. C. AND RoOSE, M. L. 1987. Patterns of genotypic diversity in clonal plant species. Am. J. Bot., 74, 123-131.

GROTH, J. V. AND ALEXANDER, H. M. 1989. Genetic divergence in spatially-separated pathogen populations. In: Jeger, M. J. (ed.) Spatial Components of Plant Disease Epidemics, pp. 165-181. Prentice-Hall, Englewood Cliffs, NJ.

GROTH, J. V., McCAJN, J. W. AND ROELFS, A. P. 1993. Intrafield founder effects for virulence in bean rust. Abstracts 6 th International Congress of Plant Pathology, p. 167. Montreal, Canada.

GROTH, J. V. AND ROELFS, A. P. 1982. Effect of sexual and asexual reproduction on race abundance in cereal rust fungus populations. Phytopathology, 72, 1503-1507.

GROTH, J. V. AND ROELFS, A. P. 1987. The concept and measurement of phenotypic diversity in Puccinia graminis on wheat. Phytopathology, 77, 1395-1397.

GROTH, J. V. AND SHRUM, R. D. 1977. Virulence in Minnesota and Wisconsin bean rust collections. Plant Dis. Rep., 61, 756-760.

HAMRICK, J. L. AND GODT, M. J. W. 1981. Allozyme diversity in plant species. In: Brown, A. H. D., Clegg, M. T., Kahler, A. L. and Weir, B. S. (eds) Plant Population Genetics: Breeding and Genetic Resources. Sinauer Associates, Sunderland, MA.

HARTL, D. L. AND ClaRk, A. G. 1989. Principles of Population Genetics, 2nd edn. Sinauer Associates, Sunderland, MA. 
LINDE, D. C., GROTH, J. V. AND ROELFS, A. P. 1989. Inheritance of two phosphoglucomutase isozymes in Uromyces appendiculatus and comparison of crossing and selfing methods. $J$. Hered., 80, 337-340.

LINDE, D. C., GROTH, J. v. AND ROELFS, A. P. 1990a. The genetic basis of isozyme variation in the bean rust fungus (Uromyces appendiculatus). J. Hered., 81, 134-138.

LINDE, D. C., GROTH, J. V. AND ROELFS, A. P. 1990b. Comparison of isozyme and virulence diversity patterns in the bean rust fungus Uromyces appendiculatus. Phytopathology, 80, 141-147.

LU, TSE-HONG AND GROTH, J. v. 1987. Isozyme detection and variation in Uromyces appendiculatus. Can. J. Bot., 66, 885-890.

McCAIN, J. W., GROTH, J. v. AND ROELFS, A. P. 1992a. Inter- and intrapopulation isozyme variation in collections from sexually reproducing populations of the bean rust fungus, Uromyces appendiculatus. Mycologia, 84, 329-340.
McCAIN, J. W., GROTH, J. V. AND ROELFS, A. P. 1992b. Are asexual populations of the bean rust fungus less diverse than sexual ones? Phytopathology, 82, 1139 (abstr.).

RODRIGUEZ, C. J., Jr., BETTENCOURT, A. J. AND RIJO, L. 1975. Races of the pathogen and resistance to coffee rust. Ann. Rev. Phytopath., 13, 49-70.

ROELFS, A. P. AND GROTH, J. v. 1980. A comparison of virulence phenotypes in wheat stem rust populations reproducing sexually and asexually. Phytopathology, 70, 855-862.

SCHWARTZ, H. F., McMILLAN, M. S. AND VAUGHN, M. R. 1990. Aecial and pycnial stages of bean rust in Colorado. Plant Dis., 74, 80 (abstr.).

STAVELY, J. R. 1983. A rapid technique for inoculation of Phaseolus vulgaris with multiple pathotypes of Uromyces phaseoli. Phytopathology, 73, 676-679.

TOOLEY, P. W., FRY, W. E. AND VILLAREAL-GONZALEZ, M. J. 1985. Isozyme characterization of sexual and asexual Phytophthora infestans populations. J. Hered., 76, 431-435. 